\title{
THE KAPLANSKY CONDITION AND RINGS OF ALMOST STABLE RANGE 1
}

\author{
MOSHE ROITMAN
}

(Communicated by Irena Peeva)

\begin{abstract}
We present some variants of the Kaplansky condition for a KHermite ring $R$ to be an elementary divisor ring. For example, a commutative K-Hermite ring $R$ is an EDR iff for any elements $x, y, z \in R$ such that $(x, y)=$ $R$ there exists an element $\lambda \in R$ such that $x+\lambda y=u v$, where $(u, z)=$ $(v, 1-z)=R$.

We present an example of a Bézout domain that is an elementary divisor ring but does not have almost stable range 1 , thus answering a question of Warren Wm. McGovern.
\end{abstract}

\section{INTRODUCTION}

First we recall some basic definitions and known results.

All rings here are commutative with unity. A ring $R$ is Bézout if each finitely generated ideal of $R$ is principal.

Two rectangular matrices $A$ and $B$ in $\mathrm{M}_{m, n}(R)$ are equivalent if there exist invertible matrices $P \in \mathrm{M}_{m, m}(R)$ and $Q \in \mathrm{M}_{n, n}(R)$ such that $B=P A Q$.

The ring $R$ is $K$-Hermite if every rectangular matrix $A$ over $R$ is equivalent to an upper or a lower triangular matrix (following [9, Appendix to §4] we use the term 'K-Hermite' rather than 'Hermite' as in [8). From [8] it follows that this definition is equivalent to the definition given there. See also [5, Theorem 3]: by this theorem, a ring is K-Hermite iff for every two elements $a, b \in R$, there are elements $a_{1}, b_{1}, d \in R$ such that $(a, b)=\left(a_{1} d+b_{1} d\right)$ and $\left(a_{1}, b_{1}\right)=R$. Parentheses are used to denote the ideal generated by the specified elements.

A ring $R$ is an elementary divisor ring (EDR) iff every rectangular matrix $A$ over $R$ is equivalent to a diagonal matrix. It follows from [8] that this definition is equivalent to the definition given there.

An EDR is K-Hermite, and a K-Hermite ring is Bézout. An integral domain is Bézout iff it is K-Hermite.

By [5, Theorem 6] a ring $R$ is an EDR iff it satisfies the following two conditions:

(1) $R$ is K-Hermite;

(2) $R$ satisfies Kaplansky's condition (see $\S 2$, condition (K) below).

Received by the editors July 15, 2011 and, in revised form, November 24, 2011.

2010 Mathematics Subject Classification. Primary 13F99.

Key words and phrases. Almost stable range 1, elementary divisor ring, Kaplansky condition, K-Hermite, stable range.

Part of this work was done while the author was visiting New Mexico State University. The author thanks Bruce Olberding from this university for useful discussions and suggestions concerning this topic. 
By [4, Example 4.11], (1) $\Longrightarrow(2)$. The question in [6] whether a Bézout domain is an EDR (equivalently, whether it satisfies Kaplansky's condition) is still open. On the other hand, $(2) \Longrightarrow(1)$ (Remark 2.1 below).

In section 2. we elaborate on the Kaplansky condition.

A row $\left[r_{1}, \ldots, r_{n}\right]$ over a ring $R$ is unimodular if the elements $r_{1}, \ldots, r_{n}$ generate the ideal $R$. The stable range $\operatorname{sr}(R)$ of a ring $R$ is the least integer $n \geq 1$ (if it exists) such that for any unimodular row $\left[r_{1}, \ldots, r_{n+1}\right]$ over $R$, there exist $t_{1}, \ldots, t_{n} \in R$ such that the row

$$
\left[r_{1}+t_{1} r_{n+1}, \ldots, r_{n}+t_{n} r_{n+1}\right]
$$

is unimodular (see comments on [9, Theorem 5.2, Ch. VIII]). For background on stable range see [1, $\S 3, \mathrm{Ch} . \mathrm{V}]$.

The ring $R$ has almost stable range 1 if every proper homomorphic image of $R$ has stable range 1 (see [10]). By [10, Theorem 3.7] a Bézout ring with almost stable range 1 is an EDR. We elaborate on the almost stable range 1 condition in $\S 3$. In particular, we present an elementary divisor domain (so Bézout) that does not have almost stable range 1, thus answering the question of Warren Wm. McGovern in [10] (Example [3.3). By Remark 3.2 below, a ring of stable range 1 is of almost stable range 1 . On the other hand, $\mathbb{Z}$ is of almost stable range 1 but is not of stable range 1 . Indeed, the stable range of $\mathbb{Z}$ is 2 : clearly, there is no integer $m$ such that $2+5 m= \pm 1$. Thus sr $\mathbb{Z}>1$. On the other hand, the stable range of any Bézout domain is $\leq 2$; hence $\mathrm{sr} \mathbb{Z}=2$. More generally, the stable range of any K-Hermite ring is $\leq 2$ [11, Proposition 8]. Also by [12, Theorem 1], a Bézout ring is K-Hermite iff it is of stable range $\leq 2$.

For general background see [8], 3, $\S 6$, Ch. 3] and [10].

\section{ON ThE KAPLANSKY CONDITION}

By [5] a K-Hermite ring $R$ is an elementary divisor ring iff it satisfies Kaplansky's condition (see [8, Theorem 5.2]):

For any three elements $a, b, c$ in $R$ that generate the ideal $R$, there exist elements $p, q \in R$ so that $(p a, p b+q c)=R$.

Remark 2.1. A local ring $R$ is of stable range 1; thus $R$ satisfies Kaplansky's condition with $p=1$. If $R$ is also a Noetherian domain, then $R$ is K-Hermite iff $R$ is a principal ideal ring. Hence a Noetherian local domain that is not a principal ideal ring is of stable range 1 but is not K-Hermite.

In the proof of Lemma 2.3 below, we will use the following well-known fact:

Remark 2.2. Let $R$ be a ring, let $A$ be a matrix in $\mathrm{M}_{m, n}(R)$, let $\mathbf{r}$ be a row in $\mathrm{M}_{1, n}(R)$, and let $1 \leq k \leq n$. Then $\mathbf{r}$ belongs to the submodule of $R^{n}$ generated by the rows of the matrix $A$ iff there exists a matrix $C \in \mathrm{M}_{k, m}(R)$ such that $\mathbf{r}$ is the first row of the matrix $C A$.

Lemma 2.3. Let $A$ be a $2 \times 2$-matrix over a ring $R$, and let $\mathbf{u}$ be a unimodular row of length 2 over $R$. Then $\mathbf{u}$ belongs to the submodule of $R^{2}$ generated by the rows of $A \Longleftrightarrow$ there exists an invertible matrix $P$ so that $\mathbf{u}$ is the first row of $P A$.

Proof. ( $\Longrightarrow$ ) : By Remark 2.2, there exists a 2-row $\mathbf{r}$ over $R$ so that $\mathbf{u}=\mathbf{r} A$. Since the row $\mathbf{u}$ is unimodular, the row $\mathbf{r}$ is also unimodular. Since $\mathbf{r}$ is unimodular of 
length 2 , there exists an invertible matrix $P$ with its first row equal to $\mathbf{r}$. Thus $\mathbf{u}$ is the first row of the matrix $P A$.

$(\Longleftarrow)$ : This follows from Remark 2.2

Lemma 2.4. Let $A$ be a $2 \times 2$ matrix over a ring $R$ so that its entries generate the ideal $R$. Then $A$ is equivalent to a diagonal matrix $\Longleftrightarrow$ the submodule of $R^{2}$ generated by the rows of $A$ contains a unimodular row.

In this case $A$ is equivalent to a matrix of the form $\left(\begin{array}{ll}1 & 0 \\ 0 & *\end{array}\right)$.

Proof. ( $\Longrightarrow)$ : By assumption, $A$ is equivalent to a diagonal matrix $D=\left(\begin{array}{cc}d_{1} & 0 \\ 0 & d_{2}\end{array}\right)$, where $d_{1}, d_{2} \in R$. Since the entries of $A$ generate the ideal $R, d_{1}, d_{2}$ also generate the ideal $R$. The sum of the rows of $D$, namely $\left[d_{1}, d_{2}\right]$, is unimodular.

$(\Longleftarrow)$ : By Lemma 2.3, the matrix $A$ is equivalent over $R$ to a matrix $B$ with first row unimodular. Hence the submodule generated by the columns of $B$ contains a column of the form $\left(\begin{array}{l}1 \\ *\end{array}\right)$. By Lemma 2.3 again (for columns), we obtain that $A$ is equivalent to a matrix $\left(\begin{array}{ll}1 & r \\ * & *\end{array}\right)$. By subtracting the first column of the matrix $\left(\begin{array}{ll}1 & r \\ * & *\end{array}\right)$ multiplied by $r$ from its second column and by a similar elementary row transformation, we obtain a diagonal matrix of the form $\left(\begin{array}{ll}1 & 0 \\ 0 & *\end{array}\right)$.

Theorem 2.5 (see [8, Theorem 5.2] and [5, Corollary 5]). Let $R$ be a ring. Let $A=\left(\begin{array}{ll}a & b \\ 0 & c\end{array}\right)$ a triangular $2 \times 2$-matrix over $R$ so that $(a, b, c)=R$. Then $A$ is equivalent to a diagonal matrix over $R$ iff there exist elements $p, q$ in $R$ so that $(p a, p b+q c)=R$.

Proof. Since $p[a, b]+q[0, c]=[p a, p b+q c]$ for any elements $p, q \in R$, the theorem follows from Lemma 2.4 .

Remark 2.6. Let $R$ be any ring. If Kaplansky's condition $(p b+q c, p a)=R$ holds for elements $a, b, c, p, q \in R$, then

$$
(p b+q c, a)=(p, c)=R .
$$

Indeed, Kaplansky's condition implies that

$$
(p b+q c, a)=(p b+q c, p)=R,
$$

so $(p, c)=R$. Cf. the next proposition.

Proposition 2.7. Let $R$ be a K-Hermite ring, and let $a, b$ and $c$ be elements of $R$ that generate the ideal $R$. Then the following four conditions are equivalent:

(1) The matrix $\left(\begin{array}{ll}a & b \\ 0 & c\end{array}\right)$ is equivalent to a diagonal matrix.

(2) There exist elements $p, q$ in $R$ so that $(p a, p b+q c)=R$.

(3) There exist elements $p$ and $q$ in $R$ so that $(p b+q c, a)=(p, c)=R$.

(4) For some elements $\lambda, u, v \in R$ we have $b+\lambda c=u v$, and $(u, a)=(v, c)=R$.

Moreover, in (4) we may choose the elements $u$ and $v$ such that $(u, v)=R$.

Proof. (1) $\Longleftrightarrow(2)$ : This follows from Theorem 2.5.

$(2) \Longrightarrow(4)$ : Since $(p a, p b+q c)=R$ we obtain $R=(p, p b+q c)=(p, q c)$, so $(p,(p b+q c) c)=R$. Let $v$ be an element of $R$ so that

$$
v p \equiv 1(\bmod (p b+q c) c) ;
$$

thus $v p \equiv 1(\bmod c)$. We have $v(p b+q c) \equiv b(\bmod c)$, so $v(p b+q c)=b+\lambda c$ for some element $\lambda \in R$. Hence $b+\lambda c=u v$, where $u=p b+q c$; thus $(u, a)=(v, c)=$ $(u, v)=R$. 
(4) $\Longrightarrow(3):$ We have $b \equiv u v(\bmod c)$. Let $p \in R$ so that $p v \equiv 1(\bmod c)$. Hence $p b \equiv u(\bmod c)$, so there exists an element $q \in R$ such that $p b+q c=u$. Thus (3) holds.

$(3) \Longrightarrow(2)$ : Since $R$ is a K-Hermite ring, we may write $(d)=(p, q)$ and $d=p_{1} p+q_{1} q$ with $\left(p_{1}, q_{1}\right)=R$. Hence

$$
\left(p_{1}, p_{1} b+q_{1} c\right)=\left(p_{1}, q_{1} c\right)=R,
$$

so $\left(p_{1} a, p_{1} b+q_{1} c\right)=\left(p_{1}, c\right)=R$. Condition (2) holds with $p$ and $q$ replaced by $p_{1}$ and $q_{1}$, respectively.

In the proof of Proposition 2.7, we used the assumption that $R$ is K-Hermite just for the implication $(3) \Longrightarrow(2)$.

Remark 2.8. If $R$ is a Bézout domain, then the following condition is equivalent to the conditions of Proposition 2.7.

(*) For some elements $\lambda, a_{1}, c_{1} \in R$ we have

$$
b+\lambda c \mid\left(1-a_{1} a\right)\left(1-c_{1} c\right) .
$$

Indeed, assume condition $(*)$. Let $u \in R$ so that

$$
(u)=\left(b+\lambda c, 1-a_{1} a\right)
$$

thus $(u, a)=R$ and $\frac{b+\lambda c}{u} \mid\left(\frac{1-a_{1} a}{u}\right)\left(1-c_{1} c\right)$. Since $\left(\frac{b+\lambda c}{u}, \frac{1-a_{1} a}{u}\right)=R$, we see that $v:=\frac{b+\lambda c}{u}$ divides $1-c_{1} c$, so $(v, c)=R$. Thus condition $(*)$ implies condition (4) of Proposition 2.7 The converse implication is obvious.

Since a K-Hermite ring is an EDR iff each matrix of the form $\left(\begin{array}{ll}a & b \\ 0 & c\end{array}\right)$ with $(a, b, c)=$ (1) has a diagonal reduction [8, Proposition 2.7 provides necessary and sufficient conditions for a K-Hermite ring to be an EDR. We present an additional condition in the next proposition.

Theorem 2.9. Let $R$ be a K-Hermite ring. The following two conditions are equivalent:

(1) $R$ is an elementary divisor ring.

(2) For any elements $x, y, z \in R$ such that $(x, y)=R$, there exists an element $\lambda \in R$ such that $x+\lambda y=u v$, where $(u, z)=(v, 1-z)=R$.

Moreover, the elements $u$ and $v$ can be chosen such that $(u, v)=R$.

Proof. (1) $\Longrightarrow(2)$ [including the requirement that $(u, v)=(1)$ ]: We apply condition (4) of Proposition 2.7 to the elements $a=z, b=x, c=y(1-z)$.

$(2) \Longrightarrow(1)$ : We verify condition (4) of Proposition 2.7. Let $(a, b, c)=R$. Let $(d)=(b, c)$; thus $(d, a)=(b, c, a)=R$. Hence $a \mid 1-d_{1} d$ for some element $d_{1} \in R$. Also $b=b_{1} d, c=c_{1} d$, where $\left(b_{1}, c_{1}\right)=R$. We apply condition (2) of the present proposition to the elements

$$
x=b_{1}, y=c_{1}, z=d_{1} d .
$$

Thus there are elements $\lambda_{1}, u_{1}, v \in R$ so that $b_{1}+\lambda_{1} c_{1}=u_{1} v$, where $\left(u_{1}, 1-d_{1} d\right)=$ $\left(v, d_{1} d\right)=R$. Let $u=d u_{1}$; thus $(u, a)=1$. Let $\lambda=\lambda_{1} d$. Hence $b+\lambda c=$ $d\left(b_{1}+\lambda_{1} c_{1}\right)=u v$ and $(u, a)=R$. We have $(v, c)=\left(v, d c_{1}\right)=\left(v, c_{1}\right)$ since $(v, d)=R$. Since $v$ divides $b_{1}+\lambda_{1} c_{1}$, it follows that $\left(v, c_{1}\right) \mid b_{1}$, so $\left(v, c_{1}\right)=R$. Thus $(v, c)=R$, as required. 
Proposition 2.10. Let $R$ be a Bézout domain. The following two conditions are equivalent:

(1) $R$ is an elementary divisor ring.

(2) For any nonzero elements $x, y, z \in R$, there exist elements $\lambda, a, b \in R$ such that $x+\lambda y \mid y(1-a z)(1-b(1-z))$ in $R$.

Proof. $(1) \Longrightarrow(2)$ : Let $(d)=(x, y)$; thus $\frac{x}{d}$ and $\frac{y}{d}$ are comaximal. By Theorem [2.9] there are elements $\lambda, a, b \in R$ so that $\left(\frac{x}{d}+\lambda \frac{y}{d}\right) \mid(1-a z)(1-b z(1-z))$. Hence $x+\lambda y \mid d(1-a z)(1-b(1-z))$, so $x+\lambda y \mid y(1-a z)(1-b(1-z))$.

$(2) \Longrightarrow(1)$ : Let $x_{0}$ and $y_{0}$ be comaximal elements in $R$, and let $z \in R$. Thus $\left(x_{0}+\lambda y_{0}\right) \mid y_{0}(1-a z)(1-b(1-z))$ for some elements $\lambda, a, b \in R$. Since the elements $x_{0}+\lambda y_{0}$ and $y_{0}$ are comaximal, we obtain that $\left(x_{0}+\lambda y_{0}\right) \mid(1-a z)(1-b(1-z))$, so $R$ is an EDR by Remark 2.8 .

\section{ON Rings OF ALmost Stable RANGe 1}

Proposition 3.1. Let $R$ be any ring. The following conditions are equivalent:

(1) $R$ is of almost stable range 1.

(2) For each nonzero element $z \in R$, the ring $R / z R$ is of stable range 1 .

(3) For every three elements $x, y, z \in R$ such that $(x, y)=R$ and $z \neq 0$, there exists an element $\lambda \in R$ such that $(x+\lambda y, z)=R$.

Proof (Cf. [1, Proposition 3.2, Ch. V]).

$(1) \Longrightarrow(2) \Longrightarrow(3)$ : Clear.

$(3) \Longrightarrow(1)$ : Let $I$ be a nonzero ideal of $R$ and let $z$ be a nonzero element in $I$. Let $x+I, y+I$ be two comaximal elements in $R / I$. Hence there exist elements $r, s \in R$ such that $1-r x-s y \in I$. By assumption, there exists an element $\lambda \in R$ such that $(x+\lambda(1-r x), z) R=R$. Thus $x+\lambda s y$ is invertible modulo the ideal $I$. It follows that $R / I$ is of stable range 1 , so $R$ is almost of stable range 1 .

Remark 3.2. The implication $(3) \Longrightarrow(1)$ in the previous proposition is clear since if $T$ is a homomorphic image of a ring $R$ with finite stable range, then $\operatorname{sr}(T) \leq \operatorname{sr}(R)$ [1. Proposition 3.2, Ch. V], although this fact was not used explicitly but rather its proof (in the above proof of the implication $(2) \Longrightarrow(3)$ we have $\operatorname{sr}(R / I) \leq$ $\operatorname{sr}(R /(z))=1)$. This fact implies that if $R$ is an arbitrary ring of stable range 1 , then $R$ is of almost stable range 1, thus answering the question in [10, Remark 3.3]. See also [10, Proposition 3.2].

As we have seen in $₫ 2$, the stable range 1 property implies Kaplansky's condition for an arbitrary ring. The converse is false since even if $R$ is an elementary divisor domain so that $R$ satisfies Kaplansky's condition, $R$ does not necessarily have almost stable range 1 .

Example 3.3. An elementary divisor domain (and so Bézout) that does not have almost stable range 1 (this example answers the question in [10, Remark 4.7]).

We use a well-known example of a Bézout domain, namely, $R=\mathbb{Z}+X \mathbb{Q}[X]$ (for a general theorem on pullbacks of Bézout domains, see [7, Theorem 1.9]). $R$ is an elementary divisor ring by [2, Theorem 4.61]. However, $R / X \mathbb{Q}[X]$ is isomorphic to $\mathbb{Z}$ and sr $\mathbb{Z}=2$. Hence $R$ does not have almost stable range 1 . 
We conjecture that a Bézout domain that is a pullback of type $\square$ (as defined in [7) of elementary divisor domains is again an EDR. In this case the conditions of [7. Theorem 1.9] must be satisfied. If this conjecture proves to be false, this will yield a negative answer to the question in [6] as to whether a Bézout domain is an EDR.

\section{REFERENCES}

[1] Hyman Bass, Algebraic K-theory, W. A. Benjamin, Inc., New York-Amsterdam, 1968. MR0249491 (40:2736)

[2] Douglas Costa, Joe L. Mott, and Muhammad Zafrullah, The construction $D+X D_{s}[X]$, J. Algebra 53 (1978), no. 2, 423-439. MR.0506224 (58:22046)

[3] László Fuchs and Luigi Salce, Modules over non-Noetherian domains, Mathematical Surveys and Monographs, vol. 84, American Mathematical Society, Providence, RI, 2001. MR 1794715 (2001i:13002)

[4] Leonard Gillman and Melvin Henriksen, Rings of continuous functions in which every finitely generated ideal is principal, Trans. Amer. Math. Soc. 82 (1956), 366-391. MR0078980(18:9d)

[5] Some remarks about elementary divisor rings, Trans. Amer. Math. Soc. 82 (1956), 362-365. MR0078979 (18:9c)

[6] Melvin Henriksen, Some remarks on elementary divisor rings. II, Michigan Math. J. 3 (1955), 159-163. MR0092772 (19:1155i)

[7] Evan Houston and John Taylor, Arithmetic properties in pullbacks, J. Algebra 310 (2007), no. 1, 235-260. MR2307792 (2008b:13028)

[8] Irving Kaplansky, Elementary divisors and modules, Trans. Amer. Math. Soc. 66 (1949), 464-491. MR0031470(11:155b)

[9] T. Y. Lam, Serre's problem on projective modules, Springer Monographs in Mathematics, Springer-Verlag, Berlin, 2006. MR2235330 (2007b:13014)

[10] Warren Wm. McGovern, Bézout rings with almost stable range 1, J. Pure Appl. Algebra 212 (2008), no. 2, 340-348. MR2357336 (2008h:13033)

[11] Pere Menal and Jaume Moncasi, On regular rings with stable range 2, J. Pure Appl. Algebra 24 (1982), no. 1, 25-40. MR647578 (83g:16025)

[12] B. V. Zabavs'kil, Reduction of matrices over Bezout rings of stable rank at most 2, Ukraïn. Mat. Zh. 55 (2003), no. 4, 550-554. MR2072558

Department of Mathematics, University of Haifa, Mount Carmel, Haifa 31905, Israel E-mail address: mroitman@math.haifa.ac.il 